\title{
AN INFINITE LOOP SPACE MACHINE FOR THEORIES WITH NONCONTRACTIBLE MULTIPLICATION
}

\author{
IGOR KRIZ \\ (Communicated by Thomas Goodwillie)
}

\begin{abstract}
In this paper, we develop a new kind of infinite loop space machine starting with theories with possibly noncontractible multiplication. As an application, we give an example of an infinite loop space machine with 'coherent' homotopy inverses.
\end{abstract}

Schwänzl and Vogt [12] recently showed that if one wants to parametrize both the multiplication and the homotopy inverses in infinite loop spaces by the same 'theory' (for a definition, see $\S 1.1$ ), then the parametric spaces of the multiplication cannot be contractible. But the contractibility of these spaces is an intuitively motivated requirement, as it encodes the higher commutativities of the infinite loop space structure. Also, it is a property which made the passage from homotopy coherent to strict data possible in many cases (see, e.g., [11, 14]). One may therefore tend to conclude from [12] that homotopy inverses simply cannot be a part of the structure of an infinite loop space machine.

The purpose of this note, however, is to show that the last conclusion is, in effect, false. We construct an infinite loop space machine that accepts theories in which multiplication may be parametrized by a noncontractible space or there may even be no subspace specifically parametrizing multiplication (see Theorem 1.3). Instead, the theory must satisfy an alternate requirement (see (1.3.1)). The theories associated with $E_{\infty}$-operads satisfy both our requirement and the contractibility requirement [12, Example 2.4]. By a result of May and Thomason [8], in this case, our machine and the machines of Boardman and Vogt [1], May [4], and Segal [13] turn out equivalent spectra. Our machine, however, accepts theories of a more general nature, including theories which also encode homotopy inverses. By [12], of course, such theories cannot satisfy the contractibility requirement of [12, Definition 2.3].

As an application of our machine, we give a criterion for a self-functor on the category of based topological spaces to be weakly equivalent to $Q(Q X=$ $\Omega^{\infty} \Sigma^{\infty} X$; see Theorem 1.5).

Received by the editors February 24, 1992 and, in revised form, July 29, 1992.

1991 Mathematics Subject Classification. Primary 55P47, 18C10.

Key words and phrases. Infinite loop spaces, theories.

The author is supported in part by the NSF. 


\section{Statement of the Results}

1.1. Preliminaries. Throughout this note, we shall work in the base category $\mathscr{T}$ of nondegenerately based compactly generated weak Hausdorff spaces. One may also consider $\mathscr{T}$ as a topological category where the object space is discrete and the homs are given the compact-open topology made compactly generated. We can then speak of continuous functors between $\mathscr{T}$ and another topological category.

Equivalence means weak homotopy equivalence. We recall some basic facts about monads and theories, essentially following Boardman and Vogt [1].

The theory of finite based sets $\vartheta_{S}$ has object set $\mathbf{N}$ consisting of the finite sets $\mathbf{n}=\{0,1, \ldots, n\}$ based at $0(n \geq 0)$. Morphisms from $\mathbf{m}$ to $\mathbf{n}$ in $\vartheta_{S}$ are based maps from $\mathbf{n}$ to $\mathbf{m}$ (contravariantly). Note that a based space $X$ specifies a functor $R X: \vartheta_{S} \rightarrow \mathscr{T}$ which is given on objects by $(R X)_{n}=X^{n}$ and on morphisms by the obvious maps (diagonals and inclusions of the base point). The category $\vartheta_{S}$ is isomorphic to the category $\Gamma$ introduced by Segal [13]. The opposite category $\left(\vartheta_{S}\right)^{\mathrm{Op}}$ is denoted by $\mathscr{F}$ [8].

A based theory is a topological category $\vartheta$ with object space $\mathbf{N}$ and an inclusion $\vartheta_{S} \hookrightarrow \vartheta$ such that $\prod_{i=1}^{n}\left(l_{i},-\right): \vartheta(\mathbf{m}, \mathbf{n}) \rightarrow \vartheta(\mathbf{m}, \mathbf{1})^{\mathbf{n}}$ is the identity where $l_{i} \in \vartheta_{S}(\mathbf{n}, \mathbf{1})$ is the map in $\vartheta_{S}$ given by $l_{i}(1)=i$ and $l_{i}(0)=0$.

A $\vartheta$-space is a based space $X$ together with a functor $A: \vartheta \rightarrow \mathscr{T}$ such that the composition $\vartheta_{S} \hookrightarrow \vartheta \stackrel{A}{\longrightarrow} \mathscr{T}$ is equal to $R X$. Note that the category of $\vartheta_{S}$-spaces is canonically isomorphic to $\mathscr{T}$; in the sequel, these two categories will sometimes be identified.

Also recall that a monad in $\mathscr{T}$ is a functor $D: \mathscr{T} \rightarrow \mathscr{T}$ together with natural transformations $\mu: D D \rightarrow D$ (composition) and $\eta:$ Id $\rightarrow D$ (unit) such that the obvious associativity and unit diagrams commute (see, e.g., [4, Chapter 1]). A $D$-space is a based space together with an action map $\lambda: D X \rightarrow X$ such that the obvious associativity and left unit diagrams commute (see [4, Chapter 1]). A $D$-functor, on the other hand, is a functor $F: \mathscr{T} \rightarrow$ Cat where Cat is an arbitrary category together with a natural transformation $\rho: F D \rightarrow F$ ("right $D$-action") such that, again, the obvious associativity and right unit diagrams commute.

Boardman and Vogt [1, Chapter 2] established passages both ways between monads theories. For a continuous monad $D$, we can talk about the theory of $D$-spaces $\vartheta_{D}$ where $\vartheta_{D}(\mathbf{m}, \mathbf{n})=D(\mathbf{m})^{n}$. Thus, an element of $\vartheta_{D}(\mathbf{m}, \mathbf{n})$ is a mapping $\mathbf{n} \rightarrow D(\mathbf{m})$. This map and the monad composition $\mu$ give rise to a composition map

$$
\vartheta_{D}(\mathbf{n}, \mathbf{p}) \times \vartheta_{D}(\mathbf{m}, \mathbf{n}) \rightarrow \vartheta_{D}(\mathbf{m}, \mathbf{p}) .
$$

Moreover, the identity $\mathbf{I d} \in \vartheta_{D}(\mathbf{n}, \mathbf{n})=D(\mathbf{n})^{n}$ has coordinates $\operatorname{Id}_{i}=\eta_{\mathbf{n}}(i)$ (recall that $\eta_{X}: X \rightarrow D X$ is the unit of the monad).

Conversely, to a theory $\vartheta$, one can associate a monad $D_{\vartheta}$. We first recall an auxilliary construction. For functors $X: \vartheta_{S} \rightarrow \mathscr{T}$ and $Y: \mathscr{F} \rightarrow \mathscr{T}$ we can define a 'coend'

$$
Y \times_{\vartheta_{S}} X=\coprod\left(Y_{m} \times X_{m}\right) / \sim,
$$

where $\sim$ is the equivalence relation generated by $(y \sigma, x) \sim(y, \sigma x)$ 
for $x \in X_{m}, y \in Y_{n}, \sigma \in \vartheta_{S}(\mathbf{m}, \mathbf{n})$. For a based theory $\vartheta$ and a $\vartheta_{S}$-space $X$, put

$$
\left(D_{\vartheta} X\right)_{n}=\vartheta(-, \mathbf{n}) \times_{\vartheta_{S}} X .
$$

By Lemma 2.2, $D_{\vartheta}$ is a monad in $\vartheta_{S}$-spaces (which is the same as a monad in $\mathscr{T})$.

Note that these definitions make sense more generally. For a general functor $D: \mathscr{T} \rightarrow \mathscr{T}$, not necessarily a monad, we define a functor $\vartheta_{D}: \mathscr{F} \rightarrow \mathscr{T}$ by

$$
\vartheta_{D}(\mathbf{m})=D(\mathbf{m}) \text {. }
$$

For a general functor $\vartheta: \mathscr{F} \rightarrow \mathscr{T}$ we define a functor $D_{\vartheta}: \mathscr{T} \rightarrow \mathscr{T}$ by

$$
D_{\vartheta} X=\vartheta(-) \times_{\vartheta_{S}} X \text {. }
$$

1.1.1. Theorem (Boardman-Vogt [1, Chapter 2]). For a theory $\vartheta, \vartheta_{D_{\theta}} \cong \vartheta$. Moreover, the categories of $\vartheta$-spaces and $D_{\vartheta}$-spaces are isomorphic via a canonical isomorphism which is the identity on objects.

(The first sentence of this theorem is also true for an arbitrary functor $\vartheta: \mathscr{F} \rightarrow \mathscr{T})$.

Proof. The first statement follows from Lemma 2.1 below. For the second statement, note that $\vartheta(-, \mathbf{n}) \times_{\vartheta_{S}}-$ is left adjoint to the forgetful functor from $\vartheta$-spaces to $\vartheta_{S}$-spaces (it is the left Kan extension). Our statement therefore follows from the characterization of algebras over adjunction monads.

The relationship between $D_{\vartheta_{D}}$ and $D$ for a functor $D$ is more subtle. We can define a canonical map $\varepsilon: D_{\vartheta_{D}} \rightarrow D$ as follows: We have

$$
D_{\vartheta_{D}}(X)=\coprod\left(D(\mathbf{n}) \times X^{n}\right) / \sim .
$$

Define the map on each $D(\mathbf{n}) \times X^{n}$ as the adjoint

$$
\varepsilon_{n}: D(\mathbf{n}) \times \mathscr{T}(\mathbf{n}, X) \rightarrow D X
$$

of the obvious map $\nabla_{n}: \mathscr{T}(\mathbf{n}, X) \rightarrow \mathscr{T}(D \mathbf{n}, D X)$ (given just by applying the functor $D$ ).

But this brings up trouble: $\varepsilon_{n}$, and therefore $\varepsilon$, may not be continuous. For example, if $D: \mathscr{T} \rightarrow \mathscr{T}$ is defined by assigning to a topological space $X$ the discrete set $X$, then $D_{\vartheta_{D}}(X)=X$ and

$$
\varepsilon=\mathrm{Id}: X \rightarrow \text { discrete } X \text {. }
$$

The map $\varepsilon_{n}$ is continuous if all the maps $\nabla_{n}$ are continuous which, in turn, happens if $D: \mathscr{T} \rightarrow \mathscr{T}$ is a continuous functor. (Recall that for a monad $D$, we had to assume that the maps $\nabla_{n}$ are continuous, anyway, to get a continuous composition on $\vartheta_{D}$. .) A functor $D$ is called finitary if

(1.1.2) The map $\varepsilon: D_{\vartheta_{D}} \rightarrow D$ is an isomorphism of functors.

Since every functor of the form $D_{\vartheta}$ is obviously continuous, we see that every finitary functor is continuous. 
1.1.3. Example. If $\mathscr{C}$ is an operad, then the corresponding monad $C$ is finitary.

We next state our results on connections between monads, theories, and infinite loop spaces. We recall the notion of an $\mathscr{F}$-space from May and Thomason [8]. First call a functor $X: \mathscr{F} \rightarrow \mathscr{T}$ proper if

(1.1.4) for any injection $\varphi \in \mathscr{F}(\mathbf{m}, \mathbf{n}), X(\varphi)$ is a $\Sigma_{\varphi}$-equivariant cofibration where $\Sigma_{\varphi}$ is the group of permutations $\sigma: \mathbf{n} \rightarrow \mathbf{n}$ such that $\sigma(\operatorname{Im} \varphi)=$ $\operatorname{Im} \varphi$.

An $\mathscr{F}$-space is a proper functor $X: \mathscr{F} \rightarrow \mathscr{T}$ such that

(1.1.5) $X_{0}$ is aspherical (i.e., equivalent to a point) and

(1.1.6) for $n>1$, the map $X_{n} \rightarrow X_{1}^{n}$ with coordinates $\delta_{i}$ is an equivalence where $\delta_{i}: \mathbf{n} \rightarrow 1$ is the map in $\mathscr{F}$ given by $\delta_{i}(j)=1$ if $i=j$ and $\delta_{i}(j)=0$ otherwise.

(Observe that $\delta_{i}$ may also be viewed as a map $\mathbf{1} \rightarrow \mathbf{n}$ in $\vartheta_{S}$. .) If we drop the properness requirement, such functors $X: \mathscr{F} \rightarrow \mathscr{T}$ are called improper $\mathscr{F}$-spaces. By [8, Appendix B], for every improper $\mathscr{F}$-space $X$ there is a functorial $\mathscr{F}$-space $X^{\prime}$ together with a natural equivalence $X^{\prime} \rightarrow X$.

1.2. Theorem. Let $D$ be a monad in $\mathscr{T}$ such that

(1.2.1) the canonical map $D(X \vee Y) \rightarrow D X \times D Y$ is an equivalence for any pair of based spaces $X, Y$,

(1.2.2) $D(*) \rightarrow *$ is an equivalence.

Then any $D$-space is canonically equivalent to an $\mathscr{F}$-space. (More precisely, there is a functorial $\mathscr{F}$-space $\underline{X}$ and a natural equivalence $\underline{X}_{1} \rightarrow X$.)

1.3. Theorem. Let $\vartheta$ be a theory such that

(1.3.1) $\prod_{i=1}^{m}\left(-, \delta_{i}\right): \vartheta(\mathbf{m}, \mathbf{1}) \rightarrow \vartheta(\mathbf{1}, \mathbf{1})^{m}$ is an equivalence for all $m \geq 0$.

Then any $\vartheta$-space is canonically equivalent to an $\mathscr{F}$-space.

1.4. Examples. If $\mathscr{D}$ is an $E_{\infty}$-operad, then the theory $\vartheta_{D}$ is an $E_{\infty}$-theory in the sense of Schwänzl and Vogt [12]. The theory $\vartheta_{D}$ also satisfies the hypothesis (1.3.1). In effect, the map of (1.3.1) is the canonical map $D(\mathbf{m}) \rightarrow D(\mathbf{1})^{m}$, which, if $\mathscr{D}$ is an $E_{\infty}$-operad, is just a model for the canonical map

$$
\coprod_{n_{1} \cdots n_{m} \geq 0} B\left(\Sigma_{n_{1}} \times \cdots \times \Sigma_{n_{m}}\right) \rightarrow\left(\coprod_{n \geq 0} B\left(\Sigma_{n}\right)\right)^{m},
$$

which is easily seen to be an equivalence. (Here we use the convention $\Sigma_{0}=$ $\Sigma_{1}=\{1\}$, which is, after all, consistent with the definition of the factorial.)

Thus, Boardman and Vogt [1] and Theorem 1.3 give two different ways of constructing a spectrum (or an infinite loop space) from the theory $\vartheta_{D}$. Both ways are equivalent by the results of May and Thomason [8].

Consider now the monad $Q\left(Q X=\Omega^{\infty} \Sigma^{\infty} X\right) . Q$ is not finitary, but it is known that the canonical map $D_{\vartheta_{Q}}(X) \rightarrow Q(X)$ is an equivalence, at least if the space $X$ is sufficiently nice $[13,3]$. The theory $\vartheta_{Q}$ satisfies $(1.3 .1)$, so we can reconstruct the infinite loop space structure from $\vartheta_{Q}$-spaces by a conceptual procedure, without utilizing the "geometrical" structure of $Q$ (Theorem 1.3). Moreover, $\vartheta_{Q}$ clearly contains homotopy inverses so that every $\vartheta_{Q}$-space is 
a grouplike $\mathscr{F}$-space, i.e., an infinite loop space. Note that, in accordance with the notation of Schwänzl and Vogt [12], there is also an augmentation $\vartheta_{Q} \rightarrow \mathscr{C} \mathscr{G}$ where $\mathscr{C} \mathscr{G}$ is the theory of abelian groups. Schwänzl and Vogt's theorem [12, Theorem 3.1] implies that the fibre $G^{-1}\left(\lambda_{n}\right)$, where $\lambda_{n}$ is the $n$ fold multiplication, cannot be contractible. In effect, $G^{-1}\left(\lambda_{n}\right) \sim\left(Q_{1}\right)^{n}$ where $Q_{1}$ is the connected component of $Q S^{0}$ consisting of maps of degree 1 .

One may object that the theory $\vartheta_{Q}$ is not very useful, because it is too large. There is a nice geometrical model of $\vartheta_{Q}$ due to Caruso and Waner [2]. The functor $\widetilde{C}_{n}$ constructed in [2, pp. 150-151] has a natural structure of a monad. Moreover, one has stabilization maps $\widetilde{C}_{n} \rightarrow \widetilde{C}_{n+1}$ (a multiplication by $I$ ). Passage to a colimit (cofibration properties are good enough so we do not need to use a telescope) gives a finitary monad $\widetilde{C}$ together with an explicit equivalence $\widetilde{C} \rightarrow Q$. The associated theory $\vartheta_{\widetilde{C}}$ is the desired approximation of $\vartheta_{Q}$. Theorem 1.2 suggests the question as to how general are the monads satisfying Condition 1.2.1. Even if we add the hypothesis that homotopy inverses exist, there are still examples very different from $Q$. We have, however, the following theorem. Call a functor $F: \mathscr{T} \rightarrow \mathscr{T}$ proper if $\vartheta_{F}: \mathscr{F} \rightarrow \mathscr{T}$ is proper.

1.5. Theorem. A proper finitary functor $F: \mathscr{T} \rightarrow \mathscr{T}$ is weakly equivalent to $Q$ if and only if it satisfies the following conditions:

(1.5.1) The canonical map $F(X \vee Y) \rightarrow F X \times F Y$ is an equivalence.

(1.5.2) The 'product' $F X \times F X \leftarrow F(X \vee X) \stackrel{F \nabla}{\longrightarrow} F X$ has a weak homotopy inverse map $F X \rightarrow F X$.

(1.5.3) There is an element $\eta \in F\left(S^{0}\right)$ with the property that for each $k \geq 0$ there is an $n \geq 0$ such that if $X$ is n-connected, then the map $H_{*} X \rightarrow$ $H_{*} F X$ induced from the composition

$$
X=\mathscr{T}\left(S^{0}, X\right) \rightarrow \mathscr{T}\left(F\left(S^{0}\right), F(X)\right) \stackrel{\text { evaluation at } \eta}{\longrightarrow} F(X)
$$

is an isomorphism in degree $<n+k$.

\section{Proofs of the Results}

It wili be clearer to assume, in this section, that the monads $D$ from the statement of Theorem 1.2 turn cofibration maps into cofibration maps and that the functors $\mathscr{F} \rightarrow \mathscr{T}$ associated with the theories from the statement of Theorem 1.3 are proper. These hypotheses may be eliminated by the techniques of May [4], May and Thomason [8], and Segal [13].

We shall first need a few lemmas about functors $\vartheta: \mathscr{F} \rightarrow \mathscr{T}$ and the associated finitary functors $D_{\vartheta}$.

2.1. Lemma. For a functor $\vartheta: \mathscr{F} \rightarrow \mathscr{T}, D_{\vartheta}(\mathbf{m})=\vartheta(\mathbf{m})$.

2.2. Lemma. Let, for functors $\vartheta^{\prime}, \vartheta^{\prime \prime}: \mathscr{F} \rightarrow \mathscr{T}, \vartheta=\vartheta^{\prime} \times \vartheta^{\prime \prime}$. Then $D_{\vartheta} X=$ $D_{\vartheta^{\prime}} X \times D_{\vartheta^{\prime \prime}} X$.

Proof. The map $D_{\vartheta} X \rightarrow D_{\vartheta^{\prime}} X \times D_{\vartheta^{\prime \prime}} X$ is defined by

$$
\left(\left(t^{\prime}, t^{\prime \prime}\right), x\right) \mapsto\left(t^{\prime}, x\right) \times\left(t^{\prime \prime}, x\right)
$$


where $t^{\prime} \in \vartheta^{\prime}(\mathbf{n}), t^{\prime \prime} \in \vartheta^{\prime \prime}(\mathbf{n})$, and $x \in X^{n}$. The map in the opposite direction is defined by

$$
\begin{aligned}
& \left(t^{\prime},\left(x_{1}, \ldots, x_{n}\right)\right) \times\left(t^{\prime \prime},\left(y_{1}, \ldots, y_{m}\right)\right) \\
& \quad \mapsto\left(\left(t^{\prime} \pi^{\prime}, t^{\prime \prime} \pi^{\prime \prime}\right),\left(x_{1}, \ldots, x_{n}, y_{1}, \ldots, y_{m}\right)\right)
\end{aligned}
$$

where $\pi^{\prime} \in \vartheta_{S}(\mathbf{m}+\mathbf{n}, \mathbf{n})$ and $\pi^{\prime \prime} \in \vartheta_{S}(\mathbf{m}+\mathbf{n}, \mathbf{m})$ are the projections to the first and last coordinates. The two maps are inverse to each other because of the identification by $\vartheta_{S}$.

For $\vartheta \in \vartheta_{S}(\mathbf{n}, \mathbf{k})$, define $\vartheta \imath \mathbf{m} \in \vartheta_{S}(\mathbf{n m}, \mathbf{k m})$ to operate on $m$-size blocks in the same way that $\varphi$ does on points. More precisely, put

$$
(\varphi \text { اm })^{\mathrm{Op}}((i-1) m+j)=\left(\left(\varphi^{\mathrm{Op}}(i)-1\right) m+j\right) \text { for } 1 \leq i \leq k, 1 \leq j \leq m \text {. }
$$

Let $\vartheta: \mathscr{F} \rightarrow \mathscr{T}$. Define $\underline{\vartheta}_{m}: \mathscr{F} \rightarrow \mathscr{T}$ by putting $\underline{\vartheta}_{m}(\mathbf{n})=\vartheta(\mathbf{n m})$ and $\underline{\vartheta}_{m}(\varphi)=\vartheta(\varphi$ m $)$.

2.3. Lemma. $D_{\underline{\vartheta}_{m}}(X) \cong D_{\vartheta}(X \wedge \mathbf{m})$. (Note that $X \wedge \mathbf{m}=X \vee \cdots \vee X$ (m times $)$.) Proof. A map $\sigma: D_{\vartheta}(X \wedge \mathbf{m}) \rightarrow D_{\underline{\vartheta}_{m}}(X)$ is defined as follows: Let

$$
x=\left(x_{1}, \ldots, x_{n}\right) \in(X \wedge \mathbf{m})^{n}, \quad x_{i} \neq * .
$$

Define a map $\sigma_{x}: \mathbf{n} \rightarrow \mathbf{n m}$ by $\sigma_{x}(j)=(j-1) m+i$ if $x_{j} \in l_{i} X$ where $l_{i}: X \rightarrow X \wedge \mathbf{m}$ is the $i$ th injection. Let, further, $\nabla: X \wedge \mathbf{m} \rightarrow X$ be the codiagonal. For $d \in \vartheta_{n}$, let

$$
\sigma:(d, x) \mapsto\left(d \sigma_{x}, \nabla^{n} x\right)
$$

The inverse map $\tau: D_{\underline{\vartheta}_{m}}(X) \rightarrow D_{\vartheta}(X \wedge \mathbf{m})$ is defined as follows. Let $e \in \vartheta_{n m}$, $x=\left(x_{1}, \ldots, x_{n}\right) \in X^{n}$. Put $\bar{x}=\left(l_{1} x_{1}, \ldots, l_{m} x_{1}, \ldots, l_{1} x_{n}, \ldots, l_{m} x_{n}\right)$,

$$
\tau:(e, x) \mapsto(e, \bar{x})
$$

Define a map $\lambda \in \vartheta_{S}(\mathbf{n}, \mathbf{n m})$ by $\lambda((i-1) m+j)=i, 1 \leq i \leq m, 1 \leq j \leq n$. Compute

$$
\begin{aligned}
\tau \sigma(d, x) & =\left(d \sigma_{x}, l_{1} \nabla x_{1}, \ldots, l_{m} \nabla x_{1}, \ldots, l_{1} \nabla x_{n}, \ldots, l_{m} \nabla x_{n}\right) \sim(d, x), \\
\sigma \tau(e, x) & =\left(e \sigma_{\bar{x}}, x_{1}, \ldots, x_{1}, \ldots, x_{n}, \ldots, x_{n}\right) \\
& =\left(e \sigma_{\bar{x}}, \lambda\left(x_{1}, \ldots, x_{n}\right)\right) \sim\left(e \sigma_{\bar{x}}(\lambda / \mathbf{m}), x\right)=(e, x)
\end{aligned}
$$

(as $\sigma_{\bar{x}}(\lambda l \mathbf{m})=1_{n}$ in $\left.\vartheta_{S}\right)$.

2.4. Proof of Theorem 1.2. Put, for $X \in \operatorname{Obj} \mathscr{T}, \underline{D}_{n} X=D(X \wedge \mathbf{n})$. Then $\underline{D} X$ is an $\mathscr{F}$-space via the action of $\mathscr{F}$ on the $\mathbf{n}$-coordinate. To see that $D(X \wedge \mathbf{n}) \rightarrow$ $(D X)^{n}$ is an equivalence, simply note that $X \wedge \mathbf{n}=X \vee \cdots \vee X(n$ times) and use condition (1.2.1). Now note that $\underline{D}_{m}: \mathscr{T} \rightarrow \mathscr{T}$ is a $D$-functor. To define the action $\underline{D}_{m} D X \rightarrow \underline{D}_{m} X$, first corisider an injection $l_{i}: X \rightarrow X \wedge \mathbf{m}$. This injection induces a map $D\left(l_{i}\right): D(X) \rightarrow D(X \wedge \mathbf{m})$. Summing these maps gives a map

$$
D\left(l_{1}\right) \vee \cdots \vee D\left(l_{m}\right): D(X) \wedge \mathbf{m} \rightarrow D(X \wedge \mathbf{m}) .
$$

We now define the action $\underline{D}_{m} D X \rightarrow \underline{D}_{m} X$ to be the composition

$$
\underline{D}_{m} D X=D(D X \wedge \mathbf{m}) \underset{D\left(D \imath_{1} \vee \cdots \vee D l_{m}\right)}{\longrightarrow} D D(X \wedge \mathbf{m}) \rightarrow D(X \wedge \mathbf{m})=\underline{D}_{m} X .
$$


The proof of the associativity of the action is easily reduced to the commutativity of the diagram:

$$
\begin{array}{cc}
D D X \wedge \mathbf{m} \quad \underset{D l_{1} D \vee \cdots \vee D l_{m} D}{\longrightarrow} & D(D X \wedge \mathbf{m}) \\
D D l_{1} \vee \cdots \vee D D l_{m} \backslash & \mid D\left(D l_{1} \vee \cdots \vee D l_{m}\right) \\
D D(X \wedge \mathbf{m})
\end{array}
$$

This diagram commutes when precomposed with either of the injections $l_{i} D D$ be the functoriality of $D$ and therefore commutes.

Now, since each $\underline{D}_{m}: \mathscr{T} \rightarrow \mathscr{T}$ is a $D$-functor, $(\underline{D}: \mathscr{T} \rightarrow \mathscr{F})$-spaces is a $D$-functor. Let, for a $D$-space $X$,

$$
\underline{X}=B(\underline{D}, D, X) .
$$

Then $\underline{X}$ is an $\mathscr{F}$-space, and the collapse map

$$
\underline{X}_{1}=B(D, D, X) \rightarrow X
$$

is an equivalence. Moreover, $B(\underline{D}, D, X)$ is an $\mathscr{F}$-space since it is the geometric realization of a simplicial $\mathscr{F}$-space.

We now turn to the proof of Theorem 1.3. Note that, in view of the proof of Theorem 1.2, it would be sufficient to show that (1.3.1) implies that $D_{\underline{\vartheta}}(X \wedge \mathbf{m}) \rightarrow\left(D_{\vartheta} X\right)^{m}$ is an equivalence. However, we do not know how to prove this. By Lemmas 2.2 and 2.3, it would be sufficient to know that if $\vartheta \rightarrow \vartheta^{\prime}$ is an equivalence of functors $\mathscr{F} \rightarrow \mathscr{T}$ then the induced map $D_{\vartheta} X \rightarrow D_{\vartheta} X$ is an equivalence. This, however, is false; the issue is the lack of a freeness condition on the actions of $\Sigma_{n} \subseteq \mathscr{F}$.

To prove Theorem 1.3, we use simplicial sets. Recall (from May [7]) that the singular set functor $S$ from spaces to simplicial sets is right adjoint to the realization functor $T$. Analogously, $S$ and $T$ are functors between based spaces and simplicial based sets and are still adjoint (because the unit and counit are based maps). On occasion, we shall also use $T$ to denote the realization of simplicial spaces (in the cases when the symbol $\|$ is too awkward).

2.5. Lemma. Let $\vartheta_{1} \rightarrow \vartheta_{2}$ be an equivalence of functors $\mathscr{F} \rightarrow \mathscr{T}$. Then, for any based set $S$, the induced map

$$
D_{\vartheta_{1}} S \rightarrow D_{\vartheta_{2}} S
$$

is an equivalence.

Proof. For a compact space $K$, a map $\varphi: K \rightarrow D_{\vartheta_{i}} S$ factors through $D_{\vartheta_{i}} L$ for some finite subset $L \subseteq S$. The map

$$
D_{\vartheta_{1}} L \rightarrow D_{\vartheta_{2}} L
$$

is an equivalence by Lemma 2.1 and the hypothesis.

2.6. Lemma. For a simplicial space $A, D_{\vartheta} T A=T\left(D_{\vartheta}\right)_{*}(A)$.

Proof. Compute

$$
D_{\vartheta} T A=\theta \times_{\theta_{S}} R T A \cong \theta \times_{\theta_{S}} T R_{*} A \cong T\left(\theta \times_{\theta_{S}} R_{*} A\right)=T\left(D_{\vartheta}\right)_{*}(A) .
$$


2.7. Lemma. Let $\vartheta: \mathscr{F} \rightarrow \mathscr{T}$ be a proper functor. Then, for any simplicial set $A, \underline{D}_{\theta} T A$ is an $\mathscr{F}$-space.

Proof. Lemma 2.3 asserts that $\underline{D}_{\vartheta_{n}}=D_{\underline{\vartheta}_{n}}$, and Lemma 2.2 asserts that $D_{\left(\vartheta^{n}\right)}=$ $\left(D_{\vartheta}\right)^{n}$. We need to show that the map

$$
D_{\underline{\vartheta}_{n}} T A \rightarrow\left(D_{\vartheta}\right)^{n} T A
$$

(as in the first paragraph of 2.4) is an equivalence. Using Lemma 2.6, all we need to show is that

$$
T D_{\underline{\vartheta}_{n}} A \sim \tilde{\rightarrow} T\left(\left(D_{\vartheta}\right)^{n} A\right) .
$$

But $D_{\vartheta_{n}} A \rightarrow\left(D_{\vartheta}\right)^{n} A$ is a simplicial equivalence by Lemma 2.5 .

2.8. Proof of Theorem 1.3. By Lemma 2.7, for a $\vartheta$-space $X$ we may put

$$
X=B\left(D_{\vartheta} T, S D_{\vartheta} T, S X\right) \text {. }
$$

We now turn to the proof of Theorem 1.5. There is a map

$$
\lambda: \Sigma^{n} F X \rightarrow F \Sigma^{n} X
$$

given as follows: For $s \in S^{n}, a \in\left(\theta_{F}\right)_{n}$, and $x=\left(x_{1}, \ldots, x_{n}\right) \in X^{n}$, put

$$
\lambda(s \wedge(a, x))=\left(a,\left(s \wedge x_{1}, \ldots, s \wedge x_{n}\right)\right)
$$

(observe that the base points are $\mathrm{OK}$, since choosing $s=0$ or $x=*$ gives $*$ on both sides).

2.9. Lemma. Under the assumptions (1.5.1) and (1.5.2) of Theorem 1.5, the composition

$$
F X \stackrel{\eta F}{\longrightarrow} \Omega^{n} \Sigma^{n} F X \stackrel{\Omega^{n} \lambda}{\longrightarrow} \Omega^{n} F \Sigma^{n} X
$$

is an equivalence.

Proof. Put $\vartheta=\vartheta_{F}$. By Lemma 2.3,

$$
D_{\underline{\vartheta}_{m}} X \cong F(X \wedge \mathbf{m}),
$$

and therefore, by condition (1.5.1), $D_{\left.\underline{\vartheta}_{-}\right)} X$ is an $\mathscr{F}$-space. Segal gives a canonical map

$$
\Sigma^{n} F X \rightarrow B_{n} F X=D_{\underline{\theta}_{(-)}} X \times_{\theta_{S}} R\left(S^{n}\right)
$$

(see [13, Proposition 3.2]) whose adjoint

$$
F X \rightarrow \Omega^{n} \Sigma^{n} F X \rightarrow \Omega^{n}\left(D_{\underline{\vartheta}_{(-)}} X \times_{\theta_{S}} R\left(S^{n}\right)\right)
$$

is an equivalence (by condition (1.5.2) and [13, Proposition 1.5]). Now, by [13, Lemma 3.7], $D_{\underline{\vartheta}_{(-)}} X \times_{\theta_{S}} R\left(S^{n}\right)$ is canonically identified with $F \Sigma^{n} X$, with a commutative diagram of canonical maps:

$$
\begin{gathered}
\Sigma^{n} F X \\
\downarrow \searrow_{\lambda}^{\lambda} \\
D_{\underline{\vartheta}_{(-)}} X \times_{\theta_{S}} R\left(S^{n}\right)=F \Sigma^{n} X
\end{gathered}
$$

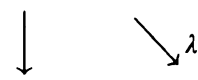

Our statement follows. 
2.10. Proof of Theorem 1.5. Necessity is obvious, as the conditions (1.5.1)(1.5.3) are satisfied by $Q$ (even though $Q$ is not a finitary functor). Conversely, let (1.5.1)-(1.5.3) hold. Denote the natural transformation $(\eta,-): I_{d} \rightarrow F$ induced by the element $\eta \in\left(\theta_{F}\right)_{1}$ by $u$. Note that the following diagrams commute:

$(2.10 .1)$

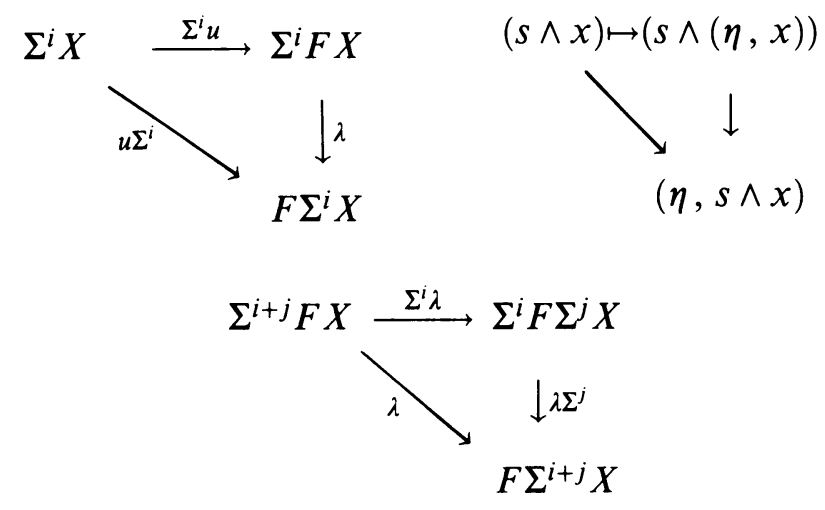

$(2.10 .2)$

$$
\left(s^{i} \wedge s^{j} \wedge\left(a_{k},\left(x_{1}, \ldots, x_{k}\right)\right)\right) \mapsto\left(s^{i} \wedge\left(a_{k}, s^{j} \wedge x_{1}, \ldots, s^{j} \wedge x_{k}\right)\right)
$$

On the element level, our notation is $s^{i} \in S^{i}, s^{j} \in S^{j}, a \in\left(\theta_{F}\right)_{k}$, and $x=$ $\left(x_{1}, \ldots, x_{k}\right) \in X^{k}$. Now by Lemma 2.9 , we have a map

$$
\Omega^{n} \Sigma^{n} X \stackrel{\Omega^{n} u \Sigma^{n}}{\longrightarrow} \Omega^{n} F \Sigma^{n} X \stackrel{\sim}{\sim} F X .
$$

Moreover, these maps stabilize via a diagram

$$
\begin{array}{rrr}
F X \stackrel{\Omega^{n} u \Sigma^{n}}{\sim} & \Omega^{n} F \Sigma^{n} X & \Omega^{n} \Sigma^{n} X \\
& \Omega^{n} \eta F \Sigma^{n} \downarrow & \downarrow \Omega^{n} \eta \Sigma^{n} \\
& \Omega^{n+1} \Sigma F \Sigma^{n} X \stackrel{\Omega^{n+1} \Sigma u \Sigma^{n}}{\longleftarrow} & \Omega^{n+1} \Sigma^{n+1} X \\
& \downarrow \Omega^{n+1} \lambda \Sigma^{n} X & \swarrow \Omega^{n+1} u \Sigma^{n+1} \\
& \Omega^{n+1} F \Sigma \Sigma^{n} X &
\end{array}
$$

The top rectangle commutes by the naturality of the transformation $\Omega^{n} \eta: \Omega^{n} \rightarrow$ $\Omega^{n+1} \Sigma$. The lower triangle commutes by the commutativity of $(2.10 .1)$ for $i=1$. The left-hand triangle is adjoint to $(2.10 .2)$ for $i=1, j=n$.

We now conclude our proof by showing that $\Omega^{n} u \Sigma^{n}$ is a $k$-equivalence for sufficiently large $n$. Diagram $(2.10 .1)(i=n)$ by adjunction gives rise to a 
commutative diagram:

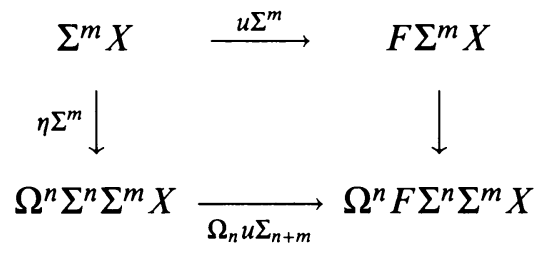

By assumption 1.5.3, if $m$ is sufficiently large, the top row induces an isomorphism in homology in degrees $\leq m+k$. The right-hand column is an equivalence by Lemma 2.9. The left-hand column is an isomorphism in degrees $\leq m+k$ if $n$ is sufficiently large. We conclude that if $m, n$ are sufficiently large, the bottom row is an $(m+k)$-equivalence. and, therefore, $\Omega^{m+n} u \Sigma^{m+n}: \Omega^{m+n} \Sigma^{m+n} X \rightarrow \Omega^{m+n} F \Sigma^{m+n} X$ is a $k$-equivalence.

\section{ACKNOWLEDGMENT}

I am very indebted to $\mathrm{J}$. P. May for valuable discussions.

\section{REFERENCES}

1. J. M. Boardman and R. M. Vogt, Homotopy invariant algebraic structures on topological spaces, Lecture Notes in Math., vol. 347, Springer, Berlin, 1973.

2. J. Caruso and S. Waner, An approximation to $\Omega^{n} \Sigma^{n} X$, Trans. Amer. Math. Soc. 265 (1981), 147-162.

3. I. Kriz, The infinite loop spaces of Thom spectra, J. Pure Appl. Algebra 81 (1992), 49-62.

4. J. P. May, The geometry of iterated loop spaces, Lecture Notes in Math., vol. 271, Springer, Berlin, 1972.

5. ___ $E_{\infty}$ spaces, group completions and permutative categories, London Math. Soc. Lecture Note Ser., vol. 11, Cambridge Univ. Press, Cambridge and New York, 1974.

6. __ Multiplicative infinite loop space theory, J. Pure Appl. Algebra 26 (1982), 1-69.

7. __ Simplicial objects in algebraic topology, Van Nostrand, New York, 1967.

8. J. P. May and R. Thomason, The uniqueness of infinite loop space machines, Topology 17 (1978), 205-224.

9. J. Milnor, The realization of a semi-simplicial complex, Ann. of Math. (2) 65 (1957), 357362.

10. D. Quillen, Higher algebraic $K$-theory. I, Lecture Notes in Math., vol. 341, Springer, Berlin, 1973, pp. 85-147.

11. R. Schwänzl and R. M. Vogt, Homotopy ring spaces and their matrix rings, Proceedings of the Poznan 1989 Algebraic Topology Conference, Lecture Notes in Math., vol. 1474, Springer-Verlag, New York, 1991, pp. 254-272.

12. __. $E_{\infty}$-Monoids with coherent homotopy inverses are abelian groups, Topology 28 (1989), 481-484.

13. G. Segal, Categories and cohomology theories, Topology 13 (1974), 293-312.

14. R. J. Steiner, Infinite loop structures on the algebraic K-theory of spaces, Math. Proc. Cambridge Philos. Soc. 90 (1981), 85-111.

Department of Mathematics, The University of Chicago, 5734 South University AvenUe, Chicago, IlLinoIs 60637 